\title{
A spindle cell anaplastic pancreatic carcinoma with rhabdoid features following curative resection
}

\author{
TOMOYUKI ABE ${ }^{1}$, HIRONOBU AMANO ${ }^{1}$, KEIJI HANADA ${ }^{2}$, AKIHISA OKAZAKI $^{2}$, SHUJI YONEHARA ${ }^{3}$, \\ FUMITO KURANISHI $^{4}$, MASAHIRO NAKAHARA ${ }^{1}$, YOSHINORI KURODA ${ }^{1}$ and TOSHIO NORIYUKI ${ }^{1,5}$
}

\author{
Departments of ${ }^{1}$ Surgery, ${ }^{2}$ Gastroenterology and ${ }^{3}$ Pathology, Onomichi General Hospital, Onomichi, Hiroshima 722-8508; \\ ${ }^{4}$ Department of Surgery, Innoshima-Ishikai Hospital, Innoshima, Hiroshima 1722-2323; ${ }^{5}$ Department of Gastroenterological \\ and Transplant Surgery, Applied Life Sciences, Institute of Biomedical and Health Sciences, \\ Hiroshima University, Onomichi, Hiroshima 1734-8551, Japan
}

Received February 1, 2016; Accepted May 9, 2016

DOI: $10.3892 / \operatorname{mco} .2016 .914$

\begin{abstract}
Anaplastic pancreatic carcinoma (ANPC) accounts for $\sim 5 \%$ of all pancreatic ductal adenocarcinoma cases. Due to its rarity, its clinical features and surgical outcomes remain to be clearly understood. A 74-year-old woman was admitted to Onomichi General Hospital (Onomichi, Japan) in April 2015 without any significant past medical history. Contrast-enhanced computed tomography (CT) revealed a $9.5 \times 8.0 \mathrm{~cm}$ tumor in the body and tail of the pancreas. The patient developed acute abdominal pain 3 weeks later and the CT revealed massive abdominal bleeding caused by tumor rupture. The tumor increased in size and reached $12.0 \times 10.0 \mathrm{~cm}$ in maximal diameter. The tumor doubling time was estimated to be 13 days. ${ }^{18} \mathrm{~F}$-fluorodeoxyglucose (FDG) positron emission tomography/CT confirmed the absence of distant metastasis since FDG accumulation was detected only in the tumor lesion. Emergency distal pancreatectomy and splenectomy were performed. Histologically, the tumor was classified as a spindle cell ANPC with rhabdoid features. The patient succumbed to mortality 8 months following the surgery while undergoing systemic adjuvant chemotherapy for multiple liver metastases. ANPC is difficult to detect in the early stages due to its progressive nature and atypical radiological findings. Long-term survival can be achieved only by curative resection; therefore, surgical resection must be performed whenever possible, even if the chance of long-term survival following surgery is considered dismal. As the present case suggested, spindle cell ANPC with rhabdoid features is highly aggressive and curative-intent resection must not be delayed.
\end{abstract}

Correspondence to: Dr Hironobu Amano, Department of Surgery, Onomichi General Hospital, 1-10-23 Hirahara, Onomichi, Hiroshima 722-8508, Japan

E-mail: amanojack@star.odn.ne.jp

Key words: anaplastic carcinoma, pancreas, spindle cell type, rhabdoid features

\section{Introduction}

Since anaplastic pancreatic carcinoma (ANPC) is rare and accounts for only $2-7 \%$ of all pancreatic carcinoma cases, its clinical features and surgical outcomes remain to be elucidated $(1,2)$. A total of three pathological subtypes of ANPC exist: Spindle cell carcinoma, giant cell carcinoma, and pleomorphic carcinoma. Surgical resection is the only curative therapy for patients with ANPC since no effective systemic chemotherapy or other interventions are available; however, long-term survival remains to be achieved in patients with ANPC even following curative surgery $(1,3)$. ANPC is often associated with a delay in clinical presentation as a result of its asymptomatic nature until the tumor has progressed to an advanced stage. In several reports that have been published since the early 1900s, ANPC was referred to as 'giant-cell tumor', 'undifferentiated carcinoma' with or without osteoclast-like giant cells and 'pleomorphic carcinoma' of the pancreas (3-6).

Establishing the precise pre-operative diagnosis is difficult due to the radiological findings being atypical and similar to those of gastrointestinal stromal tumors, mucinous cyst adenocarcinomas and pancreatic carcinomas. Upon admission, the present patient's tumor was already huge and revealed enhanced rims with hypodense lesions on computed tomography $(\mathrm{CT})$ scans, which appears to be a common radiological feature of ANPCs (1).

Of the three subtypes, spindle cell carcinoma is the most aggressive subtype of sarcomatoid carcinoma and currently no effective systemic chemotherapies or other interventions are available. According to previous reports, two patients with ANPC with rhabdoid features had dismal prognoses following surgical intervention $(7,8)$. The present study described a case of spindle cell ANPC that exhibited aggressive growth following emergency curative surgery.

\section{Case report}

A 74-year-old woman without any previous medical history was admitted to our hospital in April 2015. Physical examination revealed a firm tumor located in her left upper quadrant. 


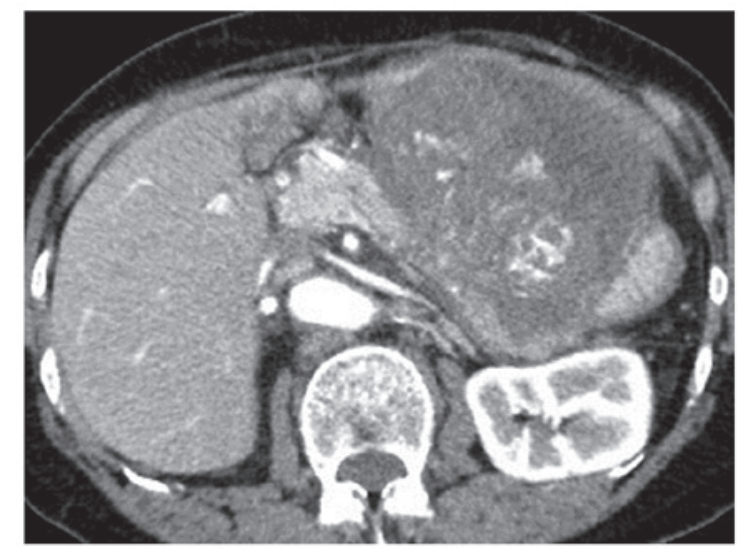

Figure 1. Abdominal computed tomography revealed a huge tumor containing a hyper-enhanced rim and heterogeneous core measuring $8.0 \times 7.5 \mathrm{~cm}$ in size.

Abdominal ultrasonography detected a giant cystic tumor containing high and low echoic lesions in the body and tail of the pancreas. Admission laboratory tests revealed that the serum levels of carbohydrate antigen 19-9, carcinoembryonic antigen and DUPAN-2 were elevated (78.0,36.6 and 1,200 U/ml, respectively). On contrast-enhanced CT scanning, the tumor occupying the left upper and lower abdomen was measured to be $9.5 \times 8.0 \mathrm{~cm}$. This tumor consisted of multilocular cystic components, both the rim and core of the tumor were strongly enhanced on the arterial phase CT images (Fig. 1). A dilatation of the distal main pancreatic duct was evident, however, no distant metastases, lymph node swelling or ascites were detected. The solid tumor exhibited low signal intensity on T1-weighted magnetic resonance imaging (MRI) and relatively high signal intensity on T2-weighted MRI (Fig. 2). Intraductal ultrasonography demonstrated a huge solid tumor derived from the body of the pancreas and located within a clear margin from the stomach (Fig. 3). ${ }^{18} \mathrm{~F}$-fluorodeoxygl ucose (FDG)-positron emission tomography/CT identified no distant metastasis, and FDG accumulation was detected only in the tumor lesion (Fig. 4). The differential diagnoses were pancreatic adenocarcinoma, gastrointestinal stromal tumor, endocrine cell carcinoma or solid pseudopapillary neoplasm of the pancreas. At 3 weeks after the initial admission of the patient, massive ascites suddenly emerged and the tumor increased in from a size of $9.5 \mathrm{~cm}$ to $11 \mathrm{~cm}$ in the maximal diameter. The patient experienced an increase in acute abdominal pain, which was uncontrollable by analgesic medications. During an emergency laparotomy, bloody ascites of 1,700 ml was observed; however, peritoneal dissemination or liver metastasis was not detected. A distal pancreatectomy and a splenectomy with regional lymph node dissection were performed. The operation lasted $4 \mathrm{~h} 13 \mathrm{~min}$. Blood loss during the operation was $1,300 \mathrm{ml}$ and the bloody ascites volume was $1,700 \mathrm{ml}$.

On macroscopic observation, the tumor was $11 \times 12 \mathrm{~cm}$, and its appearance was elastic, hard and a white mass (Fig. 5). All specimens were processed in a routine manner for paraffin embedding and $5 \mu \mathrm{m}$-thick sections were cut and stained with hematoxylin and eosin. Histopathological analysis of the sections revealed that the tumor cells had acquired dedifferentiated rhabdoid features and were positive for phosphotungstic
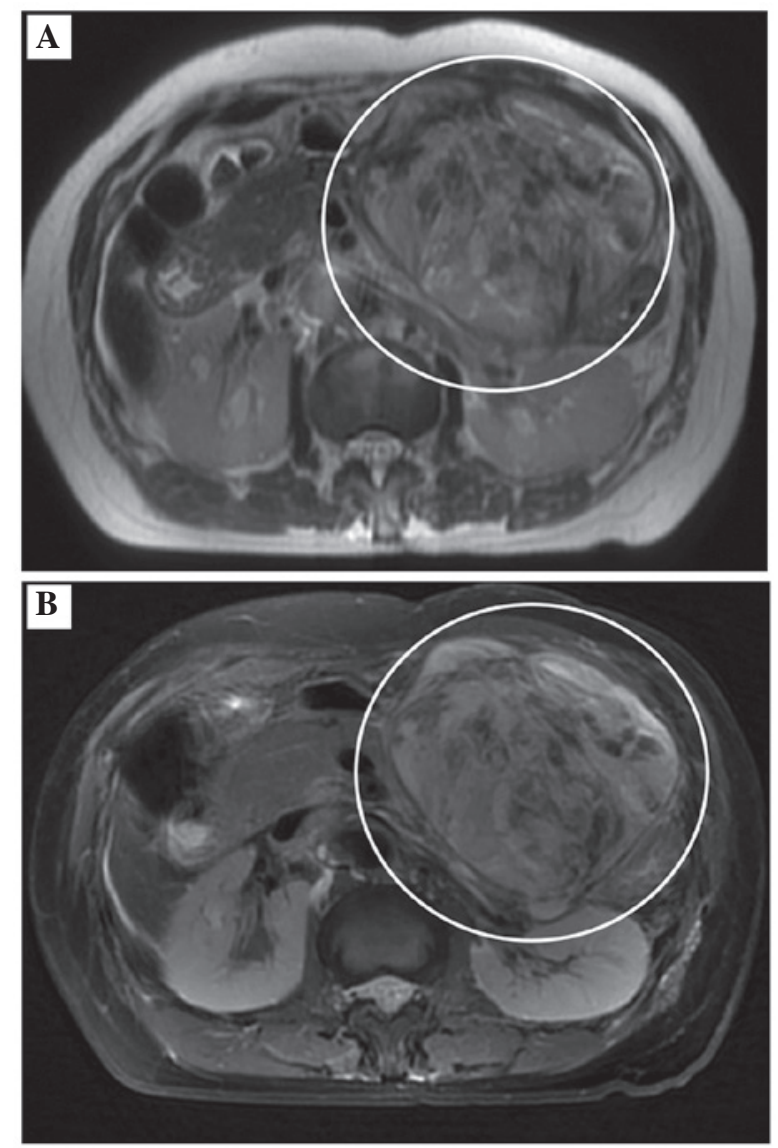

Figure 2. (A) The tumor exhibits low intensity signal on a T1-weighted MRI (B) T2-weighted MRI reveals a papillary high-intensity tumor that directly invaded the liver (white circle). MRI, magnetic resonance imaging.

acid-hematoxylin. Additionally, the tumor cells were positive for phosphotungstic acid-hematoxylin (PTAH) stain, myoglobin (cat. no. H0309; Nichilei Biosciences, Inc., Tokyo, Japan), myogenin (F5D; cat. no. 1328706B; Dako, Carpinteria, CA, USA), vimentin (V9; cat. no. H1402; Dako) and cytokeratin 5.2 (CAM52; cat. no. D04930; Roche Diagnostics, Indianapolis, IN, USA; Fig. 6). Therefore, a diagnosis of spindle cell ANPC with rhabdoid features was made based on the pathological findings. The patient was discharged 14 days post-operation without any complications. The patient succumbed to mortality 8 months following the surgery while undergoing systemic adjuvant chemotherapy.

\section{Discussion}

Spindle cell ANPC has been reported to have the worst prognosis of the three ANPC subtypes (3). The presence of rhabdoid features in ANPC is extremely rare; only two such cases have been reported to date $(7,8)$. ANPC accounts for $2-7 \%$ of all pancreatic carcinoma cases. The present case exhibited an atypical clinical presentation and radiological findings, which allowed the present study to distinguish ANPC from gastrointestinal stromal tumor, solitary pseudopapillary neoplasms and pancreatic adenocarcinoma (1). Rapid tumor progression associated with massive ascites and uncontrollable abdominal pain occurred in the present case. Distal pancreatectomy and splenectomy were successfully 


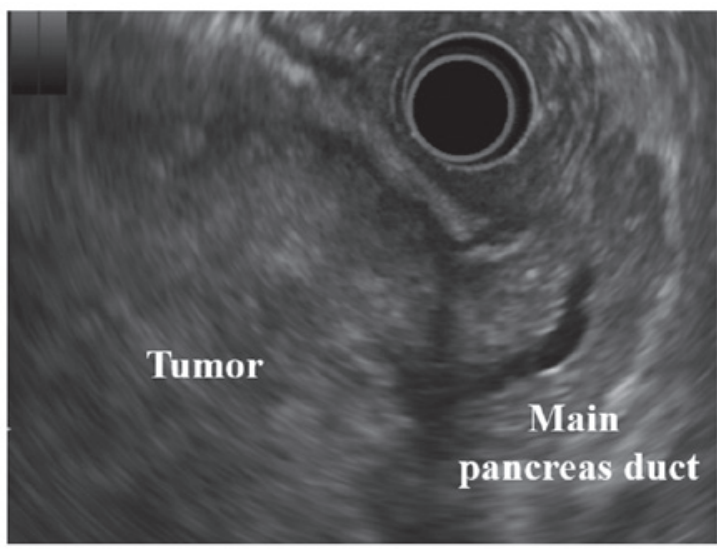

Figure 3. It was revealed that the tumor originating from the pancreatic body exhibited an irregular margin and heterogeneous enhancement. The distal main pancreatic duct was dilated due to the tumor.

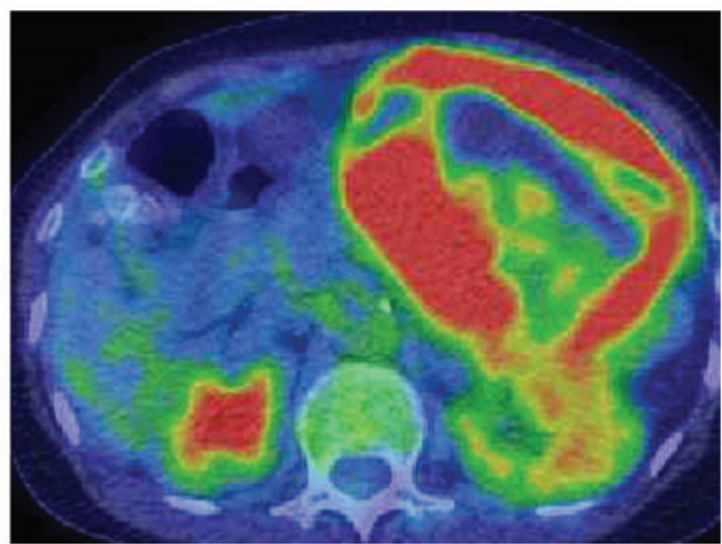

Figure 4. Positron emission tomography-computed tomography revealed no distant metastases, and ${ }^{18} \mathrm{~F}$-fluorodeoxyglucose accumulation was detected only in the tumor lesion.

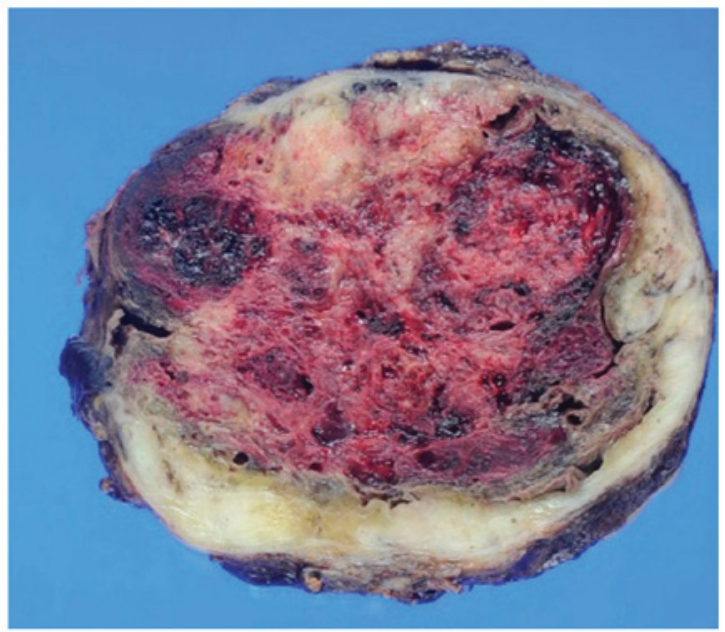

Figure 5. On macroscopic examination, the tumor is an $11 \times 12 \mathrm{~cm}$, elastic, hard, white mass.

performed to excise the giant tumor. Establishing the precise diagnosis was difficult due to the atypical radiological findings and tumor rarity. Although a huge tumor of the pancreas

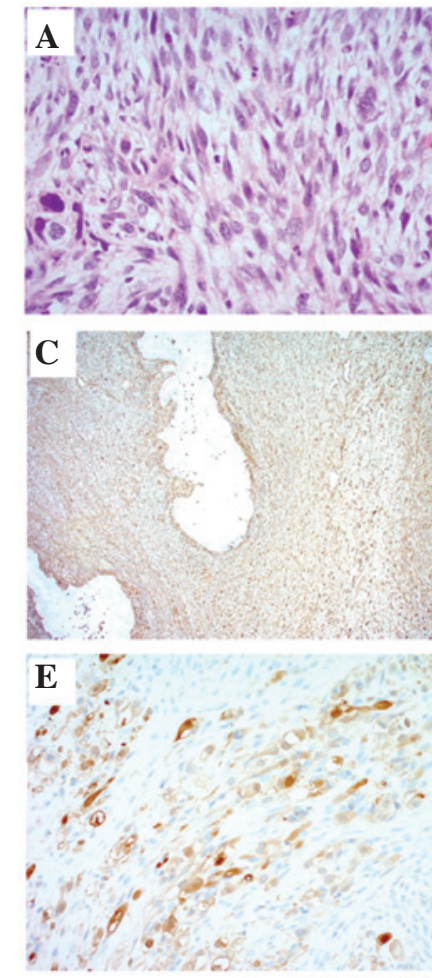

B

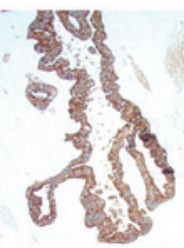

\section{央}
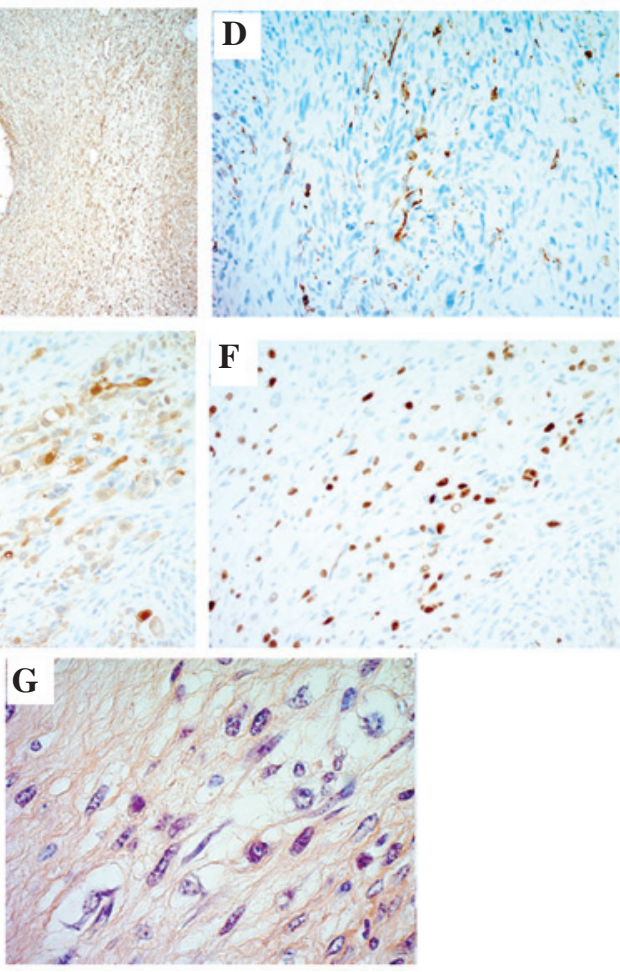

Figure 6. Histopathological phenotype of the anaplastic carcinoma with rhabdoid features. (A) The proliferation of spindle cells was observed in the stromal lesion and a papillo-tubular structure was observed in the tumor cells. (B) The tumor cells were positive for cytokeratin. (C) The tumor cells were positive for vimentin in the stromal lesion. (D) In the stromal lesion, spindle cells, which were positive for cytokeratin were observed. The tumor cells were positive for (E) myoglobin, (F) myogenin and (G) phosphotungstic acid-hematoxylin stain, and the rhabdoid features were associated with tumor cells.

can be treated via surgical resection, the prognosis is poor even following surgery. Whenever possible, radical resection is recommended.

Although multiple case reports on ANPC have been published, the clinical presentation and surgical outcomes remain to be well investigated owing to the rarity of this disease (3,9-12). Upon admission of the patient, the radiological findings on contrast-enhanced CT was a huge tumor with a well-enhanced rim and hypodense areas in the center. On T1-weighted MRI, the tumor exhibited a low signal intensity, however, on T2-weighted MRI, the intensity of the signal was high.

Strobel et al (1) reported that ANPC is an aggressive type of pancreatic cancer with a dismal prognosis (1). Whenever possible, resection must be attempted, as it is the only treatment associated with a favorable prognosis. Although the clinical presentation and radiological findings are similar to those of pancreatic carcinoma, despite the lack of a precise diagnosis, curative surgery, whenever possible, must not be postponed. 
According to previous reports, obtaining pre-operative tissue samples does not change the therapeutic approach and is not advisable if the neoplasm appears resectable.

Neither an effective chemotherapy nor a standard regimen has been established for ANPC. Neoadjuvant chemotherapy was reported to be effective in one case, however, a standard regimen was not established (13). Wakatsuki et al (12) reported that a complete response was achieved following treatment with paclitaxel (PTX) (12). The present patient was treated with a nab-PTX and gemcitabine regimen, which failed to prevent multiple live metastases and peritoneal disseminations. PTX, a microtubule-stabilizing agent, has proven effective in treating several types of cancer, including breast, lung and ovarian. PTX exerts a particularly strong antitumor activity against certain types of sarcomas including angiosarcoma, Kaposi sarcoma and carcinosarcoma of the uterus and heart (14-16).

Strobel et al (1) compared clinical features and surgical outcomes of ANPC and pancreatic ductal adenocarcinoma. In the ANPC group, the duration of survival was significantly greater following R0/R1 resection compared with after palliative treatment. On the basis of the results of the previous study, the authors recommend that patients with ANPC be operated on whenever a potentially curative resection is possible since established systemic chemotherapy or alternative therapy is available. Among the several subtypes of ANPC, the spindle cell type exhibited the worst prognosis even following curative surgery; the prognosis was particularly poor in the present case of ANPC associated with rhabdoid features.

Immunohistochemistry revealed that the present patient's tumor was positive for cytokeratin 5.2, vimentin, desmin, myoglobin, myogenin and PTAH. Kane et al (3) reviewed the immunohistochemistry results of sarcomatoid components from previous reports and their results are consistent with the present positive findings for desmin, vimentin and myogenin (3). Notably, PTAH positivity, which represents cell dedifferentiation into striated muscle, was detected only in the present case. These phenotypic changes suggested that the epithelial cancer cells had transformed into mesenchymal cancer cells. The mechanism of such a transformation may be owing to the presence of cancer stem cells or the dedifferentiation of tumor cells into sarcoma cells.

In conclusion, ANPC is a rare and aggressive variant of pancreatic cancer. Spindle cell ANPC is associated with a particularly dismal prognosis. The only typical radiological feature of ANPC is a large tumor with an enhanced rim and a hypodense area in the center. As surgical resection is the only intervention that results in long-term survival in patients with ANPC, it must be attempted whenever possible. Further studies are required to clarify the mechanism of the aggressiveness of this type of cancer.

\section{References}

1. Strobel O, Hartwig W, Bergmann F, Hinz U, Hackert T, Grenacher L, Schneider L, Fritz S, Gaida MM, Büchler MW and Werner J: Anaplastic pancreatic cancer: Presentation, surgical management, and outcome. Surgery 149: 200-208, 2011.
2. Hoorens A, Prenzel K, Lemoine NR and Klöppel G: Undifferentiated carcinoma of the pancreas: Analysis of intermediate filament profile and Ki-ras mutations provides evidence of a ductal origin. J Pathol 185: 53-60, 1998.

3. Kane JR, Laskin WB, Matkowskyj KA, Villa C and Yeldandi AV: Sarcomatoid (spindle cell) carcinoma of the pancreas: A case report and review of the literature. Oncol Lett 7: 245-249, 2014.

4. Fabre J, Planques J, Bouissou $\mathrm{H}$ and Sendrail-Pesque M: Sarcomatoid carcinoma of the pancreas. Toulouse Med 62: 85-98, 1961 (In French).

5. Alguacil-Garcia A and Weiland LH: The histologic spectrum, prognosis, and histogenesis of the sarcomatoid carcinoma of the pancreas. Cancer 39: 1181-1189, 1977.

6. Ackerman NB, Aust JC, Bredenberg CE, Hanson VA Jr and Rogers LS: Problems in differentiating between pancreatic lymphoma and anaplastic carcinoma and their management. Ann Surg 184: 705-708, 1976.

7. Kuroda N, Iwamura S, Fujishima N, Ohara M, Hirouchi T, Mizuno K, Hayashi Y and Lee GH: Anaplastic carcinoma of the pancreas with rhabdoid features and hyaline globule-like structures. Med Mol Morphol 40: 168-171, 2007.

8. Kuroda N, Sawada T, Miyazaki E, Hayashi Y, Toi M, Naruse K, Fukui T, Nakayama H, Hiroi M, Taguchi $\mathrm{H}$ and Enzan H: Anaplastic carcinoma of the pancreas with rhabdoid features. Pathol Int 50: 57-62, 2000.

9. Sano M, Homma T, Hayashi E, Noda H, Amano Y, Tsujimura R, Yamada T, Quattrochi B and Nemoto N: Clinicopathological characteristics of anaplastic carcinoma of the pancreas with rhabdoid features. Virchows Arch 465: 531-538, 2014.

10. Okazaki M, Makino I, Kitagawa H, Nakanuma S, Hayashi H, Nakagawara $H$, Miyashita T, Tajima $H$, Takamura $H$ and Ohta T: A case report of anaplastic carcinoma of the pancreas with remarkable intraductal tumor growth into the main pancreatic duct. World J Gastroenterol 20: 852-856, 2014.

11. Fujiogi M, Kobayashi T, Yasuno M and Tanaka M: Anaplastic carcinoma of the pancreas mimicking submucosal gastric tumor: A case report of a rare tumor. Case Rep Med 2013: 523237, 2013.

12. Wakatsuki T, Irisawa A, Imamura H, Terashima M, Shibukawa G, Takagi T, Takahashi Y, Sato A, Sato M, Ikeda T, et al: Complete response of anaplastic pancreatic carcinoma to paclitaxel treatment selected by chemosensitivity testing. Int J Clin Oncol 15: 310-313, 2010.

13. Jones TS, Jones EL, McManus M, Shah R and Gajdos C: Multifocal anaplastic pancreatic carcinoma requiring neoadjuvant chemotherapy and total pancreatectomy: Report of a case. JOP 14: 289-291, 2013.

14. Otsuki A, Watanabe Y, Nomura H, Futagami M, Yokoyama Y, Shibata K, Kamoi S, Arakawa A, Nishiyama H, Katsuta T, et al: Paclitaxel and carboplatin in patients with completely or optimally resected carcinosarcoma of the uterus: A phase II trial by the Japanese uterine sarcoma group and the tohoku gynecologic cancer unit. Int J Gynecol Cancer 25: 92-97, 2015.

15. Brambilla L, Romanelli A, Bellinvia M, Ferrucci S, Vinci M, Boneschi V, Miedico A and Tedeschi L: Weekly paclitaxel for advanced aggressive classic Kaposi sarcoma: Experience in 17 cases. Br J Dermatol 158: 1339-1344, 2008.

16. Ram Prabu MP, Thulkar S, Ray R and Bakhshi S: Primary cardiac angiosarcoma with good response to Paclitaxel. J Thorac Oncol 6: 1778-1779, 2011. 